\title{
LA NUOVA TRADUZIONE IN LINGUA ITALIANA DEL MESSALE ROMANO \\ L'OPPORTUNITÀ DI CELEBRARE AL PASSO COL TEMPO
}

\author{
THE NEW ITALIAN TRANSLATION OF THE ROMAN MISSAL \\ THE OPORTUNITY TO CELEBRATE IN STEP WITH TIME
}

\begin{abstract}
A b s t r a c t. This article discusses the new translation of the Roman Missal into Italian. On the first Sunday of Advent, several innovative changes were introduced for all Italian-speaking cummunities: as well as an updating of the Prayers and the Ordinary of the Holy Mass, one can also observe greater sensitivity concerning the relationship between Time and Eternity.
\end{abstract}

Keywords: Eschatology; Roman Missal in Italian; Parousia; Missale Romanum Editio Typica Tertia; New translation of the Roman Missal 2020 (KEW); Liturgy; Eucharistic celebration.

\section{INTRODUZIONE}

Un'opportunità di celebrare al passo col Tempo: non stipisca il provocatorio sottotitolo del presente articolo. Pone infatti all'attenzione del lettore la sfida che la nuova traduzione del Messale coglie: una rinnovata sensibilità

EMANUELE MiChELE DI MARCO - sacerdote, professore alla Facoltà di Teologia di Lugano, direttore del Centro Liturgico e Pastorale della Diocesi di Lugano, maestro liturgico del Vescovo. Indirizzo di corrispondenza: Corso Pestalozzi 10; CH-6900 Lugano, Svizzera; e-mail: donemanueledimarco@gmail.com

EMANUEle Michele Di MARCo - kapłan, profesor Wydziału Teologii w Lugano, dyrektor Centrum Liturgiczno-Pastoralnego diecezji Lugano, ceremoniarz liturgiczny biskupa. Adres do korespondencji: Corso Pestalozzi 10; CH-6900 Lugano, Szwajcaria; e-mail: donemanueledi marco@gmail.com 
escatologica nella Celebrazione eucaristica come risposta ad una contemporaneità sempre più contratta nel tempo immediato e disorientata ${ }^{1}$. La comunità cristiana da subito ha voluto identificarsi con la celebrazione eucaristica, ela cura verso questo memoriale - e non solo memoria ${ }^{2}$ - perpetua, nella presenza dello Spirito Santo, quel "fate questo in memoria di me" che Cristo ha affidato alla sua Chiesa. Nelle prime parole della Lettera enciclica di Giovanni Paolo II dedicata proprio al sacramento dell'Eucaristia si trova riassunto il ruolo determinante della celebrazione nella prassi della Chiesa: "La Chiesa vive dell'Eucaristia. Questa verità non esprime soltanto un'esperienza quotidiana di fede, ma racchiude in sintesi il nucleo del mistero della Chiesa"3. Dal momento dell'istituzione dell'Eucaristia fino al ritorno di Cristo alla Chiesa è affidato il compito di celebrare e di incontrare il Signore nella forma da lui stesso istituita. "Ecco, io sono con voi tutti i giorni fino alla fine del mondo" (Mt 28,20): una presenza costante che accompagna l'umanità all'incontro definitivo. La Chiesa, comunità in cammino verso il Signore risorto, celebra nel tempo in attesa dell'eternità che ne orienta i passi ${ }^{4}$. In modo particolare durante la Santa Messa si vive la tensione del "già e non ancora": "mediante la celebrazione eucaristica, ci uniamo già alla liturgia del cielo e anticipiamo la vita eterna, quando Dio sarà «tutto in tutti» (1Cor 15,

1 "Sappiamo che non basta cambiare i libri liturgici per migliorare la qualità della liturgia. Fare solo questo sarebbe un inganno. Perché la vita sia veramente una lode gradita a Dio, occorre infatti cambiare il cuore. A questa conversione è orientata la celebrazione cristiana, che è incontro di vita col «Dio dei viventi» (Mt 22,32)" (Francesco, Ai partecipanti all'assemblea plenaria della Congregazione per il Culto divino e la disciplina dei sacramenti, 14 febbraio 2019).

2 "in particolare riscoprendo i motivi delle decisioni compiute con la riforma liturgica, superando letture in- fondate e superficiali, ricezioni parziali e prassi che la sfigurano. Non si tratta di ripensare la riforma rivedendone le scelte, quanto di conoscerne meglio le ragioni sottese, anche tramite la documentazione storica, come di interiorizzarne i principi ispiratori e di osservare la disciplina che la regola. Dopo questo magistero, dopo questo lungo cammino, possiamo affermare con sicurezza e con autorità magisteriale che la riforma liturgica è irreversibile"1 (Francesco, Discorso ai partecipanti alla LXVIII Settimana Liturgica Nazionale, Roma 24 agosto 2017.)

${ }^{3}$ Giovanni Paolo II, “Enc. Ecclesia de Eucharistia, 17 aprile. 2003”, AAS 95(2003): 433475, qui 433 .

4 "Il nostro Salvatore nell'ultima Cena, la notte in cui veniva tradito, istituì il sacrificio eucaristico del suo Corpo e del suo Sangue, col quale perpetuare nei secoli, fino al suo ritorno, il sacrificio della croce, e per affidare così alla sua diletta Sposa, la Chiesa, il memoriale della sua morte e risurrezione: sacramento di pietà, segno di unità, vincolo di carità, convito pasquale, nel quale si riceve Cristo, l'anima viene ricolmata di grazia e viene dato il pegno della gloria futura". Concilio Vaticano II, "Cost. Sacrosantum Concilium", 47, AAS 56(1964): 113. 
$28)^{\prime \prime}$. In questo articolo, a partire dalla traduzione in lingua italiana del Messale Romano del 2020 (basata quindi sulla Editio typica tertia del 2002), si vuole affrontare il tema della spiritualità escatologica nella celebrazione della Messa e di come i nuovi testi in italiano assumano il compito di una più marcata catechesi sul tempo e l'eternità a partire proprio da orazioni, prefazi, preghiere. La recente traduzione, frutto di oltre diciassette anni di lavoro, non si presenta quindi come semplice adeguamento dei testi scritturistici a partire dalla nuova traduzione della Bibbia da parte della Conferenza Episcopale Italiana del 2008. Tra i vari cambiamenti subentrati a partire dalla prima domenica di Avvento del 2020, è da notare una marcata sensibilità escatologica, segno di una particolare attenzione ad un tema troppo spesso ignorato: la Chiesa deve essere orientata all'eternità ${ }^{6}$. Quand'essa dovesse smarrire il suo orientamento alla parusìa, avrebbe perso il suo senso di esistere. L'Eucaristia ha al suo centro il tempo: essa è memoria del passato (riprende le parole di Cristo stesso, pronunciate in un momento e in uno spazio ben definito), attualizzazione e presenza reale nel presente, slancio e proiezione nel futuro ${ }^{7}$. La Scrittura stessa riporta questa importante tensione nei sinottici $^{8} \mathrm{e}$ in altri testi del $\mathrm{NT}^{9}$. Essendo diverse novità, qui ci limiteremo ad una rassegna delle più rilevanti, con un'attenzione particolare al tema escatologico. La liturgia è relazione: azione del Popolo all'interno di esso, all'esterno e con Dio. È normale che una riflessione di "aggiornamento" del linguaggio rientri proprio in quest'ottica.

\section{UN PERCORSO DURATO DICIOTTO ANNI}

È del 2002 la Editio Typica tertia, segue l'edizione del 1970 e del 1975. La terza edizione del Messale Romano di Paolo VI è stata firmata da Giovanni Paolo II il 10 aprile 2000 e raccoglie un decennio di lavori: già nelle Assemblee plenarie del Dicastero competente del 1991 e del 1996 i lavori erano stati orientati in questo senso. Tra l'edizione del 1975 e quella del 2002 la differenza sostanziale è la ricezione delle normative nel frattempo pubbli-

${ }^{5}$ CCC, 1326.

6 "Fino a raggiungere la misura della pienezza di Cristo" (Ef 4,13).

${ }^{7}$ Cfr. CCC, VII. L'Eucaristia - «Pegno della gloria futura », 1402-1405.

8 "Io vi dico che d'ora in poi non berrò di questo frutto della vite fino al giorno in cui lo berrò nuovo con voi, nel regno del Padre mio" (Mt 26,29; cfr. Lc 22,18; Mc 14,25).

${ }^{9}$ Ap 1,4; 1Cor 16,22; Ap 22,20. 
cate (non è da dimenticare la pubblicazione del Codex Iuris Canonici nel 1983). Particolarmente, la possibilità di amministrare ai fedeli la comunione sotto le due specie, Tale competenza del Vescovo è primaria, conformemente a quanto stabilito dal diritto (Codice di Diritto Canonico, can. $381 \S 1$ ), per cui non è sottoposta ad una previa autorizzazione della Conferenza dei Vescovi. Inoltre, il Vescovo diocesano può rimettere la facoltà a ciascun sacerdote, in quanto pastore di una particolare comunità, il giudizio sull'opportunità di distribuzione della comunione sotto le due specie, al di fuori dei casi segnalati nei quali viene sconsigliata. Il nuovo capitolo (IX), in armonia con quanto prescritto dall'Istruzione Varietates legitimae sull'inculturazione liturgica, sottolinea una maggiore libertà della Conferenza dei Vescovi per l'introduzione nel Messale ulteriori adattamenti, che vanno considerati come particolari ed eccezionali. È stato completato il lavoro di integrazione o di adeguamento del Calendarium Romanum generale con l'inserimento di quelle celebrazioni stabilite dopo l'editio typica altera. Nell'Ordo Missae, precisamente nel corpus praefationum, è stato aggiunto un Prefazio per i martiri; il Comune della Beata Vergine Maria è stato arricchito di nuovi formulari, i cui testi sono presi per la maggior parte dei casi dalla Collectio Missarum de Beata Maria Virgine, con una migliore distribuzione degli stessi; nella sezione delle Messe ad diversasono stati inseriti due formulari particolari provenienti dal Messale preconciliare, ovvero un nuovo formulario nell'ambito delle Messe Pro remissione peccatorum, desunto dall'editio typica del 1962 dove appariva sotto il titolo Ad petendam compunctionem cordis; e il formulario della Messa ad postulandam continentiam; tra le Messe votive, poi, va segnalato l'inserimento del formulario della Messa denominata De Dei Misericordia.

Nel 2020 viene pubblicata la versione italiana del Messale del 2002, dopo un lungo lavoro di riflessione e revisione, che vede ora consegnare nelle mani della comunità uno strumento arricchito e prezioso. Affinchà questo cambiamento sia fecondo per l'intera Chiesa che celebra in lingua italiana (il riferimento è chiaramente alle comunità presenti nella Penisola, ma anche nalla Diocesi di Lugano - Svizzera - e a tutte le Missioni di lingua italiana sparse nel mondo) bisogna abbandonare l'idea del Messale come libro riservato al Clero. Il Messale è infatti, per sua definizione, il libro dell'assemblea: in esso si ritrovano le indicazioni su parole e gesti di tutta la comunità celebrante. Il rischio - realmente presente - è che la presentazione della nuova edizione italiana sia recepito nell'assemblea come un semplice adeguamento linguistico e addirittura le comunità siano rese sensibili solamente ai cambiamenti recitativi assembleari, senza alcuna coscienza di ciò che ha portato agli adeguamenti. Il pericolo è quindi di rendere le comunità semplici recettori di 
queste novità. Il principio della actuosa participatio riceverebbe un'ulteriore limitazione nella sua possibilità di realizzarsi ${ }^{10}$.

\subsection{Modifiche rilevanti}

\subsubsection{Adeguamenti alla traduzione italiana della Bibbia CEI}

Una delle modifiche più importanti e che trasversalmente coinvolge tutto il Messale è senz'altro l'adeguamento dei testi biblici. Non entriamo in merito delle singole traduzioni, rileviamo solamente che i testi approvati dalla CEI nel 2007 hanno portato cambiamenti sia nelle collette e orazioni varie, come pure nelle parti recitative del presidente ${ }^{11}$ e dell' assemblea ${ }^{12}$.

\subsubsection{Aggiornamento di alcune espressioni}

Laddove prima veniva detto semplicemente "fratelli", ora è inserita la formulazione "fratelli e sorelle"13, dando seguito ad una sensibilità contemporanea. Più curiosa è invece la modifica all'interno dei prefazi: quando si trovava l'espressione "per Cristo, nostro Signore" l'assemblea era portata talvolta a rispondere con un "amen" non previsto. Per evitare questa situazione la nuova traduzione del Messale propone "per Cristo, Signore nostro". Le preghiere eucaristiche "per varie necessità" e "della riconciliazione" trovano un linguaggio aggiornato - alcune categorie sociologiche degli anni Settanta vengono adeguate alla contemporaneità. Inoltre, "scambiatevi il dono della pace" sottolinea che la pace è ricevuta dal Signore e donata ai fratelli: non è solamente un segno, simbolo; è pace donata dallo e nello Spirito.

Assume rilevanza pure la scelta di sottolineare che l'assemblea sia radunata dallo Spirito Santo: in più occasioni la nuova traduzione utilizza queste espressioni.

${ }^{10}$ Il Concilio Vaticano II ha desiderato rendere la celebrazione eucaristica più partecipativa: sono state diverse le parti di dialogo con l'assemblea. Tuttavia si può notare che queste formulazioni liturgiche spesso non corrispondono ad una maggiore partecipazione: anzi, la memorizzazione delle stesse non giova ma anzi rischia di diventarne il limite.

11 Ad esempio: "Lavami, o Signore, dalla mia colpa, dal mio peccato rendimi puro" (lavabo, adeguato all'attuale traduzione del Sal 50).

${ }^{12}$ Ad esempio: i testi del Gloria "...e pace in terra agli uomini amati dal Signore" (differisce per motivi di cantabilità dalla traduzione della Bibbia) o del Padre Nostro "...come anche noi li rimettiamo ai nostri debitori, e non abbandonarci alla tentazione".

${ }^{13} \mathrm{Nel}$ corso del viaggio apostolico in Svizzera nel 1984, Giovanni Paolo II - a Lugano, il 13 giugno, al termine della Messa celebrata nello stadio di Cornaredo - si espresse parlando spontaneamente sottolineando che nella Messa svizzero-italiana si diceva fratelli e sorelle, al posto di solamente fratelli. Egli apprezzò particolarmente l'iniziativa dicendo che "anche a Roma si dovrebbe fare così”. 


\subsubsection{Fedeltà alla Editio typica latina}

La nuova traduzione del Messale Romano consente anche di affinare la traduzione e la fedeltà alla Editio typica latina. Ad esempio, nell'invito del sacerdote alla comunione: "Ecco l'agnello di Dio, ecco colui che toglie i peccati del mondo. Beati gli invitati alla cena dell'Agnello".

\section{TEMPO ED ESCATOLOGIA}

Si potrebbe scrivere molto sulle modifiche subentrate e soffermarsi sui singoli adeguamenti, qui si intende però soffermarsi innanzitutto sulla spiritualità escatologica che la nuova traduzione propone. Per cogliere questo importante aspetto, viene dapprima offerta una riflessione sul modo di vivere il tempo nella contemporaneità.

\subsection{La cristallizzazione del tempo}

La contemporaneità ha visto una contrazione del tempo nel presente: complici le nuove tecnologie, la caduta delle metanarrazioni, le mutate condizioni sociali e molto altro ${ }^{14}$. Tempo frammentato, informatizzato, meccanizzato, accelerato: la domanda di come l'annuncio cristiano trovi spazio in questa concezione si fa pressante. Il linguaggio ecclesiale risulta talora desueto se rapportato all'esperienza umana, in particolare modo quando si tratta di vita eterna, di regno dei cieli, di risurrezione, di Giudizio universale. Nella corsa frenetica contemporanea, più che una speranza tutto ciò sembra riferirsi a qualche pia convinzione attempata. Una società complessa, ma profondamente ferita e sebbene marcata dalla pretesa di essere autosufficiente, esprime tutta la sua vulnerabilità.

\subsection{Parusìa e vita della Chiesa}

La vita eterna è la promessa al centro dell'annuncio evangelico ${ }^{15}$ : non solo parole, con la risurrezione, il Cristo ha dimostrato di poter infrangere il

\footnotetext{
${ }^{14}$ Per un completo approfondimento della percezione del tempo nella postmodernità rinvio al mio testo: Emanuele Michele Di Marco, Il tempo si è fatto breve (Città del Vaticano: LUP, 2015).

15 «Se il cristiano non è più in grado di dare un contenuto sicuro all'espressione "vita eterna", le promesse del Vangelo, il senso della Creazione e della redenzione svaniscono, e la stessa vita presente resta priva di ogni speranza» (Sindro Maggiolini, "Domande senza risposta. Anticipazioni", in Congregazione per la Dottrina della Fede, Temi attuali di escatologia. Documenti, commenti e studi (Città del Vaticano: LE.V., 2000), 95.
} 
muro del tempo. Il mondo ha dimenticato il termine vita eterna, relegandolo nel settore del magico e del misterico ${ }^{16}$ : la comunità cristiana vive pure la medesima tentazione. Eppure, risuonano più che mai attuali le parole di Paolo VI alla riapertura del Concilio Vaticano II; il Papa si chiedeva quale fosse la missione della Chiesa e rispose alla Chiesa tutta dicendo: «un'unica risposta, che abbiamo ritenuto di doverci ribadire in quest'ora solenne e in quest'assemblea e proclamare al mondo intero: che cioè Cristo, diciamo Cristo, è il nostro principio, Cristo è la nostra guida e la nostra via, Cristo è la nostra speranza e la nostra meta» ${ }^{17}$. La vita eterna è proprio il dono che Dio offre ai suoi figli tramite Gesù Cristo: «Dio infatti ha tanto amato il mondo da dare il Figlio unigenito, perché chiunque crede in lui non vada perduto, ma abbia la vita eterna» ${ }^{18}$. La storia non è più concepita con una visione lineare, è elaborata secondo il criterio relativista. Ogni avvenimento può essere facilmente sostituito. Il tempo intermedio del già e del non ancora coinvolge quindi tanto chi sta ancora compiendo il suo cammino terreno quanto chi ha già lasciato questa vita. Il Concilio Vaticano II è stato condotto «sotto il segno del rinnovamento pastorale», e sebbene questa definizione non abbia sempre giovato ad una sua valutazione corretta, evidenziamo che la ripresa dei temi escatologici non può lasciare indifferenti. Se il Concilio è stato pastorale e ne notiamo un forte contributo sull'escatologia e la comprensione della Chiesa in rapporto ad essa, significa che tra la pastorale e l'escatologia vi è un rapporto forte, che i padri conciliari hanno voluto sottolineare e portare alla Chiesa tutta. L'indole escatologica della Chiesa si realizza nella sua semplice complessità: se a parole sembra facilmente comprensibile la proposta di recuperare questa visione unitaria a livello ecclesiologico, sul piano della realizzazione ciò richiede costanza e decisione; consente inoltre di recuperarne una visione soprannaturale e non di mera istituzione terrena, aiutando a comprendere come questa relazione permette di vedere l'azione del Cristo di continuo, come l'intercessione dei santi che continuano a sostenerla, non solamente con l'esempio, ma proprio con beni spirituali.

16 «Alla demitizzazione della terra, iniziata da Colombo, con lo sviluppo della scoperta di Copernico, la demitizzazione del cielo [...] A partire dal secolo scorso, a questa demitizzazione locale dell'immagine del mondo se ne aggiunge, in misura crescente, anche una temporale». Joseph Ratzinger, Dogma e predicazione, tr. it. (Brescia: Queriniana, 2005²), 164-165.

${ }^{17}$ Paolo VI, "Discorso di chiusura del Concilio Ecumenico Vaticano II [8 dicembre 1965]", AAS 58(1966): 7-8.

${ }^{18} \mathrm{Gv} 3,16$. 
Pure nella prassi della Chiesa dobbiamo rilevare un raffreddamento dello sguardo di fede alla morte: «va senz'altro riconosciuto che nella maggioranza dei cristiani (ci riferiamo al mondo occidentale), l'annunzio della vita eterna ("la vita del mondo che verrà" - recita l'articolo del credo niceno-costantinopolitano), non ha goduto buona fama negli ultimi decenni». Una delle mancanze nella predicazione e nella catechesi era proprio il legame tra il presente ed il futuro. Perdere il legame passato - presente - futuro ha determinato un'umanità smarrita, e questo grave difetto lo si può ritrovare anche nella pastorale.

\subsection{Eternità e prassi della Chiesa}

Per una buona ripartizione del tempo e per una irradiazione della presenza di Dio in tutta la vita, la liturgia della Chiesa si presenta come il luogo privilegiato $^{19}$, come tempo altro $^{20}$, che esprime la sua portata profetica ${ }^{21}$. In quanto azione di Cristo nella comunità che egli ha fondato, la liturgia è un luogo imprescindibile di incontro con il Signore ${ }^{22}$ : è urgente, nella contem-

${ }^{19}$ Per approfondire: Giorgio Bonaccorso, "Nuovi modelli interpretativi del tempo: provocazioni alla riflessione liturgica", Rivista liturgica 77(1990): 359-386; Silvano Maggiani, "Festa/Feste", in Domenico Sartore, Achille M. Triacca (edd.), Nuovo Dizionario di Liturgia (Cinisello Balsamo: Paoline, 1988), 555-581.

${ }^{20}$ Emblematico è l'esempio del canto del Sanctus durante la celebrazione eucaristica. Esso dev'essere vissuto come comunione con coloro che sono stati conosciuti e che, dopo essere stati chiamati dalla vita terrena, possono già partecipare del la gioia del Signore: «Nel Sanctus si uniscono la liturgia visibile e quella invisibile, la liturgia della terra e quella del cielo, la liturgia nel tempo e quella eterna. Il tempo della liturgia è luogo di salvezza perché è il tempo che va oltre se stesso e fa presentire il suo superamento [...] Emerge la dimensione escatologica del Sanctus che i padri della Chiesa hanno riconosciuto con forza. La divina liturgiya di Giovanni Crisostomo chiama il Sanctus "l'inno della vittoria"». Gofredi Boselli, Liturgia e tempo (Mignano [BI]: Qiqaion, 2012).

${ }^{21}$ Cfr. Servizio Nazionale per il progetto culturale (ed.), Il tempo della festa. Dieci voci per riscoprire la domenica (Cinisello Balsamo: San Paolo, 2005).

22 «La Chiesa celebra il mistero del suo Signore "finché egli venga" (1Cor 11,26) e "Dio sia tutto in tutti" (1Cor 15,28). Dall'età apostolica la liturgia è attirata verso il suo fine dal gemito dello Spirito nella Chiesa: "Marana tha!" (1Cor 16,22). La liturgia condivide così il desiderio di Gesù: "Ho desiderato ardentemente di mangiare questa Pasqua con voi, [...] finché essa non si compia nel regno di Dio" (Lc 22,15-16). Nei sacramenti di Cristo la Chiesa già riceve la caparra della sua eredità, già partecipa alla vita eterna, pur "nell'attesa della beata speranza e della manifestazione della gloria del nostro grande Dio e Salvatore Gesù Cristo" (Tt 2,13). "Lo Spirito e la Sposa dicono: 'Vieni!' [...]. Vieni, Signore Gesù!" (Ap 22,17.20). San Tommaso riassume così le diverse dimensioni del segno sacramentale: "Il sacramento è segno commemorativo del passato, ossia della passione del Signore; è segno dimostrativo del frutto prodotto in noi dalla sua passione, cioè della grazia; è segno profetico, che preannunzia la gloria futura”» $(C C C, 1130)$. 
poraneità, conferirle il giusto valore ${ }^{23}$. Spesso l'uomo postmoderno vede infatti nella liturgia una semplice memoria o un atto cultuale dovuto, staccandosi in tal modo da quella concezione di evento salvifico che invece le è propria. La celebrazione liturgica così non viene vissuta come importante per la propria vita e come ristoro e guida del cammino terreno di ciascuno; mentre si inserisce nel già e non ancora della Chiesa. Questa proposta sottolinea infatti l'importanza della liturgia e del tempo liturgico come relazione con il Risorto $^{24}$ : sebbene non vi sia spazio per approfondire i gesti e le parole nel loro significato profondo ed escatologico (ognuno di essi è il richiamo alla perfezione ultima e definitiva, volta a stimolare la nostalgia della patria eterna), indichiamo alcune linee di riflessione sull'importanza del tempo liturgico, che in tal modo consente di completare quanto scritto sopra nel rapporto tra il tempo lavorativo e libero e quello festivo. Non si esauriscono qui i riferimenti escatologici della liturgia. La scansione del tempo - secondo lo schema giudaico - vede la celebrazione liturgica secondo un triplo ritmo: giornaliero, settimanale, annuale. Si pone qui l'accento su alcuni elementi, tenendo conto delle emergenze indicate nel capitolo secondo: il valore della domenica, l'anno liturgico, la celebrazione della morte cristiana. Vissuta come pregustazione delle realtà definitive, la liturgia acquista un sapore unico ed un senso che non si esaurisce nell'immediato ${ }^{25}$.

23 «La presenza del Signore è dunque il primo dono da riconoscere e di cui rendere grazie. Discernerla significa riconoscere che il tempo della liturgia è luogo della salvezza perché è il tempo nel quale il Signore è in mezzo al suo popolo. Il tempo della liturgia è quel kairòs che Gerusalemme non seppe discernere e questo fu causa di pianto per il Cristo: "non hai riconosciuto il tempo (tòn kairón) in cui sei stata visitata" (Lc 19,44)». Goffredo Boselli, "Il tempo luogo della salvezza", in Aa.Vv. L'anno Liturgico. Pellegrini nel tempo. Itinerario educativo alla sequela di Cristo, Atti della LXIII Settimana Liturgica Nazionale, a cura del Centro di Azione Liturgica (Roma: Edizioni Liturgiche, 2013), 42.

24 «Quando la Chiesa celebra il Mistero di Cristo, una parola scandisce la sua preghiera: Oggi!, come eco della preghiera che le ha insegnato il suo Signore e dell'invito dello Spirito Santo. Questo "oggi" del Dio vivente in cui l'uomo è chiamato ad entrare è 1' "Ora" della Pasqua di Gesù, che attraversa tutta la storia e ne è il cardine» $(C C C, 1165)$.

25 «Il tempo è coinvolto in ciò che va oltre il tempo. Il vero atto interiore, che peraltro non avrebbe consistenza senza quello esteriore, supera il tempo, ma, poiché viene dal tempo, questo può sempre essere in esso recuperato». Joseph Ratzinger, Introduzione allo spirito della liturgia, tr. it. (Cinisello Balsamo: San Paolo, 2001), 54. 


\section{NUOVA TRADUZIONE E ESCATOLOGIA}

In un'epoca fortemente caratterizzata da una vita privata di linearità e di orizzonte ultimo, la liturgia si presenta come luogo privilegiato celebrativo e catechetico. La nuova traduzione del Messale Romano, come è stato riportato in precedenza con alcune esemplificazioni, favorisce la consapevolezza della celebrazione "hic et nunc" in attesa dell'incontro definitivo con il Signore nella parusìa. La tensione temporale verso il compimento della storia è quindi recuperato ed esplicitato. A volte si è trattato di sottolinearlo attraverso l'inserimento di un avverbio di tempo ("oggi", "ora") o di spazio ("qui", "in questo luogo") per poi esplicitare la tensione parusìaca più volte ricordata e sottolineata. È sicuramente utile a questo punto riportare alcuni esempi di questa maggiorata sensibilità, ponendo i testi del MR del 2020, ad iniziare da alcune orazioni, per poi addentrarsi nell'Ordinario della Messa (in grassetto le parti modificate nella nuova traduzione del MR):

Prima domenica di Avvento, Colletta:

Accogli, o Signore, il pane e il vino, dono della tua benevolenza, e concedi che il nostro sacrificio spirituale compiuto nel tempo sia per noi pegno della redenzione eterna.

La prima domenica di Avvento sottolinea la temporalità del sacrificio dell'Eucaristica che viene celebrata in pegno di una redenzione che viene accolta e che nella celebrazione che viene effettuata trova una prima realizzazione.

Venerdì, prima settimana di Avvento, Colletta:

Risveglia la tua potenza, o Signore, e con grande forza vieni in nostro soccorso, perché la tua grazia vinca le resistenze dei nostri peccati e affretti il momento della salvezza.

In questo caso viene sottolineata la forza dell'intervento del Redentore. Si viene salvati in un momento preciso: la pienezza del tempo, quando il Signore verrà nella gloria, qui è esplicitato in modo ancora più evidente.

21 dicembre, novena di preparazione al Natale, Colletta:

Esaudisci con bontà le preghiere del tuo popolo, o Padre, perchè coloro che si rallegrano per la venuta del tuo Figlio unigenito nella nostra carne possano giungere al premio della vita eterna quando verrà nella gloria. L'incarnazione è qui ripresa (come in altri casi) con la sottolineatura della prima venuta del Cristo, l'espressione "nella carne" vuole proprio esplicitare il mistero della Redenzione umana che inizia proprio con l'Annunciazione 
e si protrae fino alla fine dei tempi. Le due venute del Crisot sono quindi qui evidenziate maggiormente.

Epifania, Colletta:

Lo splendore della tua gloria illumini, o Signore, i nostri cuori, perchè possiamo attraversare le tenebre di questo mondo e giungere alla patria della luce senza fine.

Anche nella solennità della manifestazione del Signore il tono del proprio del giorno riprende la tensione escatologica. Essendo il tempo di Avvento per definizione il tempo che permette di rivivere il mistero dell'Incarnazione in preparazione alla seconda venuta del Cristo, non stupisce che la maggior parte delle modifiche della nuova traduzione si trovi proprio in questa sezione.

Martedì, seconda settimana di Quaresima, Sulle offerte:

Per la potenza di questo mistero di riconciliazione compi in noi, o Signore, la tua opera di salvezza, perchè ci guarisca dai mali di questo mondo e ci conduca ai beni del cielo.

Anche negli altri tempi liturgici si trova esplicitata la tensione che sottolineiamo. Anche il tempo di Quaresima consente di riprendere, in un'ottica di conversione del cuore, la fondamentale diversità tra la condizione terrena e quella celeste.

Nelle esequie fuori dal Tempo pasquale, Colletta:

O Dio, che sempre usi misericordia e perdoni, ascolta le preghiere che ti rivolgiamo per il nostro fratello [la nostra sorella] N., che [oggi] hai fatto passare a te da questo mondo, e poiché in te ha sperato e creduto, conducilo[a] alla patria del cielo e donagli [donale] la gioia senza fine.

Altri esempi si possono ritrovare nelle orazioni per le Messe dei defunti o nei formulari per varie necessità.

Di seguito sono riportati alcuni esempi tratti da dall'Ordinario della Messa.

Invito del sacerdote alla preghiera sulle offerte:

Pregate fratelli e sorelle, perché il sacrificio della Chiesa, in questa sosta che la rinfranca nel suo cammino verso la patria del cielo, sia gradito a Dio Padre onnipotente.

Per evitare la confusione con la patria terrena, la nuova traduzione del Messale esplicita "del cielo", a sottolinare quindi che il cammino nel quale si celebra l'Eucaristia è quello orientato al banchetto celeste. Stessa sensibilità 
la si ritrova nella presentazione del Corpo di Cristo dopo la litania dell'Agnello: Ecco l'Agnello di Dio, ecco colui che toglie i peccati del mondo. Beati gli invitati alla cena dell'Agnello.

Oltre all'inversione della formula (che riprende quindi la versione dell'Editio typica latina) viene richiamato che è beato non tanto chi è invitato all'Eucaristia che si sta celebrando, quanto piuttosto chi è chiamato a partecipare alla cena dell' Agnello. Il riferimento biblico al libro dell'Apocalisse $(19,2)$ è quindi evidente ed esplicito. L'Eucaristia è preludio, anticipazione del banchetto eterno e definitivo.

\section{Preghiera Eucaristica III}

... e continui a radunare intorno a te un popolo che, dall'oriente all'occidente, offra al tuo nome il sacrificio perfetto.

Più che i confini terreni, la PE III richiama il senso geografico cosmico, riprendendo tra l'altro uno squisito riferimento al ritorno di Cristo, quando egli verrà da Oriente.

...nell'attesa della sua venuta nella gloria, ti offriamo, o Padre, in rendimento di grazie, questo sacrificio vivo e santo.

La seconda venuta di Cristo sarà diversa dalla prima, sarà nella gloria. Il compimento parusìaco della storia viene qui ricordato e sottolineato.

Preghiera Eucaristica I della Riconciliazione

$\mathrm{Tu}$ che ci hai convocati intorno alla mensa del tuo Figlio, raccogli in unità gli uomini di ogni stirpe e di ogni lingua, insieme con la Vergine Maria, gloriosa Madre di Dio, con gli apostoli e tutti i santi, nel convito della Gerusalemme nuova, dove splende la pienezza della tua pace, in Cristo Gesù, Signore nostro.

Anche in questo caso si riprende l'importanza dell'Eucaristia che viene celebrata, in attesa del banchetto eterno.

\section{Preghiera Eucaristica V A}

Ora, Padre santo, celebrando il memoriale di Cristo tuo Figlio e nostro Salvatore, che per la passione e la morte di croce hai fatto entrare nella gloria della risurrezione e hai posto alla tua destra, annunciamo l'opera del tuo amore fino al giorno della sua venuta, e ti offriamo il pane della vita e il calice della benedizione. Guarda con benevolenza l'offerta della tua Chiesa: è 'il sacrificio pasquale di Cristo che egli stesso ci ha consegnato e che noi ti presentiamo. Concedi che, comunicando al suo Corpo e al suo Sangue, per 
la potenza del tuo Spirito di amore diventiamo, ora e per l'eternità, membra vive del tuo Figlio.

Le quattro preghiere eucaristiche "svizzere" trovano ora un nuovo ordine e titoli differenti. Rientrano sotto la nomenclatura "per varie necessità" e sono state riviste nelle proprie formulazioni. Qui sopra, un esempio di adeguamento.

Benedizioni solenni (Natale risp. Pasqua)

Voi, che vi rallegrate per la venuta nella carne del nostro Redentore, possiate godere la gioia della vita eterna quando egli verrà nella sua gloria. Amen.

Il Dio di ogni consolazione, che nella sua bonta' ineffabile ha creato l'uomo e nella risurrezione del suo Figlio unigenito ha concesso ai credenti la speranza di risorgere, effonda su di voi la sua benedizione. Amen. A noi, ancora pellegrini sulla terra, conceda il Signore il perdono dei peccati, e a tutti i defunti la dimora della luce e della pace. Amen. Doni a tutti noi, che crediamo in Cristo risorto dai morti, di vivere con lui nella felicita` senza fine. Amen.

\section{OPPORTUNITÀ PASTORALI}

La nuova traduzione si presenta come un'occasione particolare per celebrare ma anche riflettere e riscoprire l'attesa escatologica, troppo sovente non compresa come elemento determinante per la fede cristiana.

\subsection{Il valore della domenica}

Il giorno del Signore, dies dominica ${ }^{26}$, è il tempo di incontro con il Signore risorto $^{27}$, in particolar modo durante la Celebrazione eucaristica. Sino a che questa è vista come assolvimento del precetto non viene vissuta in tutto

${ }^{26}$ Così è chiamata per la prima volta in Ap 1,10.

${ }^{27}$ Quale esempio di fedeltà alla domenica basti riferirsi ai quarantanove martiri di Abitene, i quali, violando il divieto di celebrare l'Eucaristia, furono condannati. Alla pena capitale risposero: sine dominica non possumus. "Ohne uns am Sonntag zur Feier der Eucharistie zu versammeln, können wir nicht leben. [...] Wer den Gottesdienst als die Mitte seines Lebens erkennt, versteht sich als ein verdanktes und deshalb dankbares Lebewesen». Kurt Koch, "Die Liturgie der Kirche als Fest lebendigen Glaubens", in George Augustin, Kurt Koch, Liturgie als Mitte des christlichen Lebens (Freiburg in Br.: Herder, 2012), 28. 
il suo valore e difficilmente può risultare determinante per la vita. Il riposo del settimo giorno è santificato perché pieno della gratitudine verso il Signo$\mathrm{re}^{28}$ per i beni ricevuti.

L'incontro con il Cristo risorto avviene durante la Celebrazione eucaristi$\mathrm{ca}^{29}$, fons et culmen di tutta la vita cristiana: essa è accompagnamento e forza che accompagna la Chiesa ed ogni suo singolo membro «verso il banchetto celeste, quando tutti gli eletti si siederanno alla mensa del Regno» ${ }^{30}$, finché il Signore tornerà sulla terra ${ }^{31}$. La domenica è quindi memoria e attuazione del Mistero della risurrezione di Cristo, avvenuta il primo giorno della settimana: chiamarlo ottavo giorno significa proprio vedere in esso la nuova creazione iniziata dal Cristo ${ }^{32}$. La dimensione comunitaria della Celebrazione eucaristica, prefigurazione del banchetto escatologico per le nozze dell'Agnello $^{33}$, è significativa per la vita del cristiano perché consente di rivivere l'esperienza degli Apostoli e quali, alla sera di Pasqua, videro il Risorto $^{34}$. È una concreta risposta all'individualismo imperante della contemporaneità e anticipazione della comunione in Dio dopo la seconda venuta del Cristo. La domenica deve essere vissuta interamente come giorno del Signore, per ridarle il vero senso escatologico che le è proprio ${ }^{35}$, ma che spesso nel-

28 «La risurrezione è l'unica spiegazione plausibile della domenica. Non poteva essere scelto altro giorno e non poteva restare più il sabato, perché di domenica Cristo è risuscitato dai morti; è salito al cielo ed ancora di domici apparirà alla fine coi suoi santi angeli». Pacifico Massi, La domenica (Napoli: M. D’Auria, 1967), 43.

${ }^{29}$ «Es ist nicht nur ein festliches Miteinandersein. Was das eucharistische Mahl heilig macht, ist die Gegenwart Gottes. Wir verkünden den Tod und die Auferstehung des Herrn. In dieser Verkündigung verherrlichen wir Gott. Im verkündigten Wort wird das Heilsgeschehen heutig. Das Opfer Christi wird für unser Heil in Zeit und Raum vergegenwärtigt». George Augustin, "Die Eucharistie mit geistlichem Gewinn feiern", in Augustin, Koch, Liturgie als Mitte des christlichen Lebens, 73.

${ }^{30}$ CCC, 1344.

31 Cor 11,26 .

32 «Gesù è risorto dai morti "il primo giorno della settimana" (Mt 28,1; Mc 16,2; Lc 24,1; Gv 20,1). In quanto "primo giorno", il giorno della Risurrezione di Cristo richiama la prima creazione. In quanto "ottavo giorno", che segue il sabato, [Cfr. Mc 16,1; Mt 28,1] esso significa la nuova creazione inaugurata con la Risurrezione di Cristo. È diventato, per i cristiani, il primo di tutti i giorni, la prima di tutte le feste, il giorno del Signore (e Kyriaké eméra, "dies domini$c a$ "), la "domenica"» $(C C C, 2174)$. Gregorio di Nissa spiega l'ottavo giorno in quanto seguente al tempo diviso in sette cfr. Gregorio di Nissa, Pro Octava (PG 44, 609-612).

${ }^{33}$ Ap 19,9.

${ }^{34}$ Cfr. Gv 20,19.

35 «La domenica infatti richiama, nella scansione settimanale del tempo, il giorno della risurrezione di Cristo. È la Pasqua della settimana, in cui si celebra la vittoria di Cristo sul 
la pratica cristiana viene dimenticato. Il giorno del Signore è piuttosto da vivere come tempo in cui i cristiani siano in grado di "godere di sufficiente e riposo e tempo libero che permetta loro di curare la vita familiare, culturale, sociale e religiosa» ${ }^{36}$. Giovanni Paolo II ha dedicato una Lettera apostolica, intitolata Dies Domini, alla domenica e alla sua importanza per la vita del cristiano. Alle soglie del Grande Giubileo, il Papa ha riflettuto sulla tradizione bimillenaria della Chiesa rapportandola al contesto culturale e sociale, rilevando la preoccupante tendenza alla dimenticanza di questo giorno particolare. Il giorno ottavo, così chiamato già dai Padri, è vera figura dell'eternità. La domenica non è quindi testimone di ciclicità del tempo, ma rinvio alla centralità di Cristo nella storia. Si distingue dunque la visione cristiana della storia, grazie all'anno liturgico (particolarmente nelle domeniche).

\subsection{L'anno liturgico: orientati verso il Signore che viene}

L'anno liturgico così come è vissuto attualmente si stabilizza entro la fine del VI secolo ${ }^{37}$. Esso non è visto come un ciclico succedersi di stagioni ed avvenimenti, ma come nuova opportunità per l'irruzione dell'eterno nella storia, «la cui efficacia non può conoscere limiti di tempo o di luogo» ${ }^{38}$. Come abbiamo già rilevato, in un primo momento è la domenica, il giorno del Signore, a scandire il tempo cristiano. L'anno liturgico esprime da un lato la sua dimensione mistagogica ${ }^{39}$, celebrativa e teologica, ma dall'altro ha pure una dimensione pedagogica, che attualmente rischia di disperdersi. L'anno liturgico aiuta a rivivere quegli avvenimenti che hanno caratterizzato la vita del Cristo: dalla sua Incarnazione alla Pentecoste. Rivivendo la sua vita, si è non solo confortati ed edificati, ma pure condotti verso la risurrezione. Una vera e propria scuola di vita: l'Avvento insegna l'attesa, il Natale il

peccato e sulla morte, il compimento in lui della prima creazione, e l'inizio della "nuova creazione" (cfr. 2Cor 5,17). È il giorno dell'evocazione adorante e grata del primo giorno del mondo, ed insieme la prefigurazione, nella speranza operosa, dell' "ultimo giorno", quando Cristo verrà nella gloria (cfr. At 1,$11 ; 1$ s $4,13-17$ ) e saranno fatte "nuove tutte le cose" (cfr. Ap 21,5)» (Giovanni Paolo II, "Lettera Ap. 'Dies Domini'”, in Insegnamenti di Giovanni Paolo II, 21(1998), 1:1191. Cfr. AAS 90(1998): 714.

${ }^{36}$ Concilio Vaticano II, "Costituzione Pastorale Gaudium et Spes", 67, AAS 58(1966).

${ }^{37}$ Cfr. Aa.Vv., L'anno liturgico. Storia, teologia e celebrazione (Milano: Marietti, 1988).

${ }^{38}$ Mario Righetti, Storia liturgica, II, L'anno liturgico. Il breviario, 2 (Milano: Righetti, 1969), 6.

39 «L'anno liturgico ha quindi una dimensione mistagogica, mira cioè a introdurre i fedeli nel mistero di Cristo» (Matias Augé, "Teologia dell'anno liturgico", in PIL, Scientia liturgica, Manuale di liturgia, V, Tempo e spazio liturgico (Casale Monferrato: Piemme, 1998), 370. 
compimento delle promesse; la Quaresima come occasione di penitenza, la Pasqua la risurrezione e la conseguente gioia senza fine; oltre alle feste per varie circostanze, dogmi (Corpus Domini; Annunciazione, Immacolata Concezione e Assunzione della Beata Vergine Maria, Ascensione del Signore ${ }^{40}, .$. ) e memoria dei santi. Non solo: anche la memoria di tutti i fedeli defunti richiama al valore dell'intercessione dei viventi ${ }^{41}$. Vissuto in profonda comunione con Cristo attraverso la partecipazione alla sua vita, l'anno liturgico diviene narrazione - maturazione del rapporto con Dio: rinnovata occasione di scoprire quella vocazione che è il senso della vita di ogni uomo, l'incontro e la comunione con Dio $^{42}$. Il singolo, la società stessa non sono più i medesimi dell'anno precedente. La storia si avvicina continuamente alla sua capitolazione, per questo pare più opportuno di tempo liturgico come a spirale, piuttosto che ciclico.

Molte altre feste sono indice di speranza nella parusia ed espongono nel loro articolarsi la pedagogia dell'anno liturgico. Si intende qui sottolineare pure la solennità di Cristo Signore e re dell'universo, talora dimenticata, eppure così importante nel suo carico escatologico. Esse accompagnano il cristiano per le vie della vita, condivisa nella Chiesa $^{43}$, conferendo alle varie tappe della propria esistenza terrena un senso di speranza nel compimento delle promesse.

\subsection{Condivisione dei testi con l'assemblea}

Il Messale non è il libro del prete: è il testo della comunità dei fedeli. Cogliere l'opportunità di questo cambiamento significa condividere con tutti

40 «Agli occhi di papa Benedetto non vi è nell'anno liturgico una festa che esprima così bene l'essenza della speranza cristiana come quella dell'Ascensione di Cristo, che predice che anche la nostra propria sostanza è già arrivata presso Dio». Koch, Il mistero del granello di senape. Fondamenti del pensiero teologico di Benedetto XVI, 83.

${ }^{41}$ Ratzinger ricorda che l'uomo si comprende bene se non ci si ferma a chiedersi da dove viene, ma interrogandosi sul dove vada. Cfr. Joseph Ratzinger, Bilder der Hoffnung. Wanderungen im Kirchenjahr (Freiburg in Br.: Herder, 1997), 70.

42 «Lo scopo del tempo è questo: vivere in comunione con Dio. Il tempo ha dunque un senso preciso, perché il settimo giorno è il destino dell'uomo e di tutta la creazione: anticipazione escatologica per tutta l'umanità [...] Nell'intenzione di Dio, il tempo del credente è un tempo ritmato, un tempo altro e santo [...] è questo il senso profondo delle festività cristiane, e, attorno ad esse, dal semplice scorrere dell'anno liturgico». Enzo Bianchi, Dare senso al tempo. Le feste cristiane (Magnano: Qiqaion, 2003), 6.

43 «La Chiesa non vive che la vittoria di Cristo sulla morte, l'atto della sua Risurrezione. La vita della Chiesa non è la vita del tempo, come non è la fuga degli avvenimenti terreni la vita di ogni cristiano: la vita del cristiano e la vita di tutta la Chiesa è il mistero di Cristo». Divo Barsotti, Il mistero cristiano nell'anno liturgico (Cinisello Balsamo: San Paolo, 2004), 160. 
i fedeli le modifiche subentrate, spiegandone le motivazioni e rinnovando così il desiderio di essere sempre più una comunità che celebra in attesa dell'incontro con il Risorto.

\section{CONCLUSIONI}

Un'opportunità per celebrare al passo col Tempo: la provocatoria frase del sottotitolo ha stimolato, nel presente articolo, alla ricerca dei tratti di un rinnovato sapore escatologico all'interno della nuona traduzione del Messale Romano. In diversi ambienti ecclesiali si esige un rinnovamento di vari ambiti e settori: più che una spasmodica ricerca di qualsivoglia elemento sino a poco prima non presente. La nuova traduzione, accompagnata da una graduale coscientizzazione dei suoi cambiamenti, si inserisce nelle possibili e plausibili - risposte alle comunità e ai singoli che lamentano l'eccessiva routine delle celebrazioni.

La riforma irreversibile non potrà mai esigere il ritorno ad una forma celebrativa precedente: si tratta piuttosto di inserirsi nel cammino indicato da Cristo, Signore del tempo e della storia, che per definizione è colui che porta a compimento la Chiesa, senza retromarce o altro.

Preparazione, attuazione, espansione. Le tre dimensioni del tempo (passato, presente, futuro) si ritrovano nella gestione del tempo liturgico e della preparazione di quanto concerne l'Eucaristia.

Introduzione nel tempo di pandemia: comunità impedite a celebrare, vincolate e limitate nel respirare liturgico. Sarà appunto lui - il tempo - a confermare o meno quanto è stato qui presentato: se i rinnovati testi avranno favorito una maggiore consapevolezza dell'aspetto comunitario orientato alla parusìa, allora il lavoro compiuto avrà dato i suoi frutti.

\section{BIBLIOGRAFIA}

Concilio Vaticano II. Cost. Sacrosantum Concilium. AAS 56(1964): 97-138.

Concilio Vaticano II. Cost. Gaudium et Spes. AAS 58(1966): 1025-1115.

Paolo VI. "Discorso di chiusura del Concilio Ecumenico Vaticano II [8 dicembre 1965]". AAS 58(1966): 1-8.

Giovanni Paolo II. Enc. Ecclesia de Eucharistia, 17 aprile. 2003. AAS 95(2003): 433-475.

Congregazione per la Dottrina della Fede. Temi attuali di escatologia. Documenti, commenti e studi. Città del Vaticano: LEV, 2000. 
Francesco. Ai partecipanti all'assemblea plenaria della Congregazione per il Culto divino e la disciplina dei sacramenti, 14 febbraio 2019.

Francesco. Discorso ai partecipanti alla LXVIII Settimana Liturgica Nazionale, Roma 24 agosto 2017.

Catechismo della Chiesa Cattolica. Città del Vaticano: LEV, 1992.

Aa.Vv. L'anno liturgico. Storia, teologia e celebrazione. Milano: Marietti, 1988.

Augé, Matias. "Teologia dell'anno liturgico". In PIL. Scientia liturgica, Manuale di liturgia, V. Tempo e spazio liturgico, 370-371. Casale Monferrato: Piemme, 1998.

Barsotti, Divo. Il mistero cristiano nell'anno liturgico. Cinisello Balsamo: San Paolo, 2004.

Bianchi, Enzo. Dare senso al tempo. Le feste cristiane. Magnano: Qiqajon, 2003.

Boselli, Goffredo. Liturgia e tempo. Magnano: Qiqajon, 2012.

Boselli, Goffredo. "Il tempo luogo della salvezza". In Aa.Vv. L'anno Liturgico. Pellegrini nel tempo. Itinerario educativo alla sequela di Cristo, Atti della LXIII Settimana Liturgica Nazionale, a cura del Centro di Azione Liturgica, 40-55. Roma: Edizioni Liturgiche, 2013.

Boselli, Goffredo. "«Con la rugiada del tuo Spirito». La nuova edizione italiana del Messale romano". Rivista del Clero Italiano 101(2020), 3: 198-219.

Bonaccorso, Giorgio. "Nuovi modelli interpretativi del tempo: provocazioni alla riflessione liturgica". Rivista liturgica 77(1990): 359-386.

Di Marco, Emanuele Michele. Il tempo si è fatto breve. Città del Vaticano: LUP, 2015.

Koch, Kurt. "Die Liturgie der Kirche als Fest lebendigen Glaubens". In George Augustin, Kurt Koch. Liturgie als Mitte des christlichen Lebens. Freiburg in Br.: Herder, 2012.

Maggiani, Silvano. "Festa/Feste". In Domenico Sartore, Achille M. Triacca (ed.). Nuovo Dizionario di Liturgia, 555-581. Cinisello Balsamo: Paoline, 1988.

Massi, Pacifico. La domenica. Napoli: M. D’Auria, 1967.

Ratzinger, Joseph. Bilder der Hoffnung. Wanderungen im Kirchenjahr. Freiburg in Br.: Herder, 1997.

Ratzinger, Joseph. Introduzione allo spirito della liturgia, tr. it. Cinisello Balsamo: Paoline, 2001.

Ratzinger, Joseph. Dogma e predicazione, tr. it. Brescia: Queriniana, $2005^{2}$.

Righetti, Mario. Storia liturgica, II. L'anno liturgico. Il breviario, 2. Milano: Ancora, 1969. Servizio Nazionale per il progetto culturale (ed.). Il tempo della festa. Dieci voci per riscoprire la domenica. Cinisello Balsamo: San Paolo, 2005.

\section{LA NUOVA TRADUZIONE IN LINGUA ITALIANA DEL MESSALE ROMANO L'OPPORTUNITÀ DI CELEBRARE AL PASSO COL TEMPO}

S o m m a ri o

L'articolo parla della nuova traduzione del Messale Romano in lingua italiana. Alla prima domenica di Avvento del 2020 in tutte le comunità di lingua italiana sono entrate in vigore diverse novità: oltre ad una attualizzazione delle orazioni e dell'ordinario della Messa, si può notare una maggiore sensibilità al rapporto tempo / eternità.

Parole chiave: Escatologia; Messale Romano in lingua italiana; Parusìa; Missale Romanum Editio Typica Tertia; Nuova Traduzione; CEI Messale Romano 2020; Liturgia; Celebrazione Eucaristica. 


\section{NOWE TŁUMACZENIE MSZAŁU RZYMSKIEGO NA JĘZYK WŁOSKI}

St res z z e n i e

Artykuł dotyczy nowego tłumaczenia Mszału Rzymskiego na język włoski. W pierwszą niedzielę adwentu 2020 r. we wszystkich wspólnotach włoskojęzycznych weszły w życie różne innowacje: oprócz aktualizacji modlitw i zwykłego porządku Mszy św., daje się zauważyć większą wrażliwość na związek między czasem a wiecznością.

Słowa kluczowe: Eschatologia; Mszał Rzymski w języku włoskim; Paruzja; Missale Romanum Editio Typica Tertia; Nowe tłumaczenie Mszału Rzymskiego 2020 (KEW); Liturgia; Celebracja eucharystyczna. 